\title{
Ultraperipheral nuclear interactions
}

\author{
I.M. Dremin 1 \\ Lebedev Physical Institute, Moscow, Russia
}

Keywords: proton, nucleus, ultraperipheral interactions, cross section, form factor, impact parameter

\begin{abstract}
Large-distance ultraperipheral collisions of two relativistic ions are considered. The clouds of photons surrounding the ions are responsible for their distant electromagnetic interaction. The perturbative approach and the method of equivalent photons are described. It is shown that the total cross section of these collisions increases rapidly with increasing energy and is especially large for heavy ions. Some experimental data and their comparison with theoretical approaches are described. Further proposals are discussed.
\end{abstract}

PACS: 25.75.-q, 34.50.-s, 12.20.-m

Contents.

1. Introduction

2. The early history

3. Electron-positron pair production in ultraperipheral collisions according to the perturbation theory

3.1. The differential distributions

3.2. The bound-free processes

4. The equivalent photon approximation

5. The preasymptotical behavior of the cross sections

6. Theoretical analysis of exclusive dilepton production

7. Comparison with experimental data

8. Searches for new physics

9. Conclusions

Acknowledgements

1e-mail: dremin@lpi.ru 


\section{Introduction.}

Ultraperipheral nuclear collisions are distinguished from others by the nature of the interacting fields. They happen when the ions move not close enough to interact strongly. Then the electromagnetic fields surrounding the ions enter the game. We concentrate here on the pure ultraperipheral collisions where the photons from the two electromagnetic clouds surrounding both ions collide2. Interest in them is related to the fact that electromagnetic fields become extremely compressed in the longitudinal direction and very strong at high velocities of the ions. The cross section of these processes increases at high energies even more rapidly than the strong interaction cross section. This feature opens the way to studies of strong electromagnetic fields and their possible non-linear effects.

The peripherality of these interactions is characterized by the transverse distance between the trajectories of the centers of two colliding ions, called the impact parameter $b$. For ultraperipheral collisions $b$ must be larger than the sum of ions' radii $b>R_{1}+R_{2}$. Otherwise the ions interact strongly. The total cross sections of strong hadronic interactions at present energies are very large. The ion collisions with small impact parameters are studied, e.g., for searches of some effects due to production of the quark-gluon plasma. The mean multiplicities of particles created by strong interactions are very high. Therefore, the particles produced by ultraperipheral processes would be lost in the huge background from strong interactions. The special selection criteria dictated by the kinematics of ultraperipheral processes must be imposed to separate them. At the impact parameters, slightly exceeding the sum of the radii, the exchanged photon can excite one of the ions interacting directly with quarks and producing some bosons. The strong interactions get partly involved. The theoretical treatment becomes more complicated. That is why we will not consider such processes called as the photoproduction (or photonuclear) reactions.

The present review is rather brief. It ia aimed on those who just start to get acquainted with this problem. Its main purpose is to be a guide to the papers where the discussed problems are presented in detail. Therefore, it concentrates on some particular aspects of ultraperipheral collisions related to studies at colliders. It deals mostly with works done during the last decade

\footnotetext{
${ }^{2}$ Sometimes they are called as the two-photon processes. More photons can be involved in the interaction (radiative corrections to the two-photon graphs).
} 
with some references to the previous stages. To shorten the review, no Figures and graphs abundantly shown in many papers are demonstrated but multiple references to them are given with short resume of obtained conclusions.

We start with a brief reminder of the early history of the problem of ultraperipheral nuclear collisions. The perturbative approach to its solution is described. The asymptotical energy behavior of the cross section of the electron-positron pair production in ultraperipheral collisions is demonstrated. The higher order corrections are discussed in connection with the preasymptotical energy dependence of the cross section. The method of equivalent photons is formulated and applied to the calculation of the cross sections. The special features of the bound-free processes, where the produced electron becomes bound to one of the ions, are considered. The nuclear form factors and the suppression mechanism are discussed. Some experimental data are compared with theoretical predictions. The comparison motivates further analysis of the main assumptions used in the theoretical approaches. Searches for new physics in ultraperipheral processes are described.

\section{The early history}

Almost a century ago, in 1924, Fermi [1, 2] considered the problem of interaction of charged objects with matter: "Let's calculate, first of all, the spectral distributions corresponding to those of the electric field created by a particle with electric charge, $e$, passing with velocity, $v$, at a minimum distance, $b$, from a point, $P$." He obtained the formula for the intensity of the electromagnetic field created in this process. It was used in 1934 by Weizsäcker [3] and Williams [4] for their formulation of the method of equivalent photons as discussed below.

The same year, Landau and Lifshitz [5], impressed by the prediction of positrons in the Dirac-sea theory, used the Dirac equation and calculated the asymptotical behavior of the cross section of the production of the electronpositron pair in the electromagnetic fields of the colliding relativistic nuclei. It happened to increase very rapidly at high energies $E$ as $\ln ^{3} \gamma$ where $\gamma=$ $E / M$ is the Lorentz-factor of the colliding ions of mass $M$. That was a test for the newly born Dirac theory for the positron. It is remarkable that this paper [5] was published almost immediately after the discovery of positrons in cosmic ray interactions in 1932 (published in 1933 [6]). 
Three years later, Racah [7] got the expression for the cross section in lowest order perturbation theory (Born approximation) which contained some preasymptotical terms increasing slower than $\ln ^{3} \gamma$.

These papers gave the start for more detailed theoretical studies of such processes. Experimental research became extremely intensive after high energy colliders got into the operation.

The very careful and detailed review of theoretical predictions and some early experimental data was given in 1975 by the Novosibirsk group [8]. Let us mention also some later review papers [9, 10, 11, 12, 13, 14].

\section{Electron-positron pair production in ultra- peripheral collisions according to the per- turbation theory}

As told above, the process of the electron-positron pair production in ultraperipheral interactions of ions was the first one described theoretically. In these collisions, the two colliding protons or nuclei interact electromagnetically but not hadronically. They effectively miss each other with no change of their states. They interact only by the photon clouds, which create the electron-positron pairs. No nuclear transitions appear at small transferred momenta. The large spatial extention of electromagnetic fields and their high intensity at large velocities lead to the strong energy increase (proportional to $\ln ^{3} \gamma$ ) of the cross section of these processes. The high density of the photon clouds surrounding heavy ions is in charge of large coefficients in front of this law, proportional to the squares of their electric charges $Z_{1} e$ and $Z_{2} e$. It is $Z^{4}$ times less for the proton (the hydrogen atom nucleus!) collisions. These fields act only for a short time and the perturbation theory is appropriate. The famous Racah formula [7] for the total cross section of the ultraperipheral production of the electron-positron pair in collisions of fast nuclei derived in the Born approximation looks like

$$
\sigma_{Z_{1} Z_{2} \rightarrow Z_{1} Z_{2} e^{+} e^{-}}=\frac{28\left(Z_{1} Z_{2} \alpha^{2}\right)^{2}}{27 \pi m_{e}^{2}}\left(l^{3}-6.36 l^{2}+15.7 l-13.8\right)
$$

where

$$
l=\ln \frac{2\left(p_{1} p_{2}\right)}{M_{1} M_{2}}=\ln \frac{s_{n n}}{m^{2}}=\ln \left(4 \gamma_{c}^{2}\right)
$$


and $m_{e}$ is the electron mass, $m$ is the nucleon mass, $p_{i}$ are the 4-momenta of colliding ions with masses $M_{i}$ (considered equal on the right hand side), $\gamma_{c}$ is their Lorentz-factor in the center-of-mass system, $s_{n n}$ is the squared total energy per colliding nucleon pair. The formula contains the preasymptotical terms proportional to $l^{2}$ and $l$ slower increasing with energy increase. The small mass of the electron in the denominator favors large values of the cross section. The ultraperipheral production cross sections for heavy lepton pairs $\left(\mu^{+} \mu^{-}\right.$or $\left.\tau^{+} \tau^{-}\right)$can be obtained at relativistic energies from the Racah formula (11), which does not take into account the form factors of the colliding objects, by inserting their masses in place of the electron mass 3 . The cross sections are proportional to the inverse squares of the lepton masses and, therefore, become much smaller than those for the electron pairs.

In terms of the energy per the pair of colliding nucleons $\sqrt{s_{n n}}$ the Racah formula (1) can be rewritten as

$$
\sigma_{Z_{1} Z_{2} \rightarrow Z_{1} Z_{2} e^{+} e^{-}}=\frac{28\left(Z_{1} Z_{2} \alpha^{2}\right)^{2}}{27 \pi m_{e}^{2}}\left(\ln ^{3} \frac{s_{n n}}{8.3 m^{2}}+2.2 \ln \frac{s_{n n}}{8.3 m^{2}}+0.4\right) .
$$

This formula absorbs the strongest correction terms $l^{2}$ in Eq. (11) inside the leading term. The numerical factor in the argument of the logarithms is responsible for doing that. Therefore, this formula can be directly applied for studies of the preasymptotical behavior of the cross section. This is important in view of newly constructed NICA and FAIR facilities with energies $\sqrt{s_{n n}}$ about $10 \mathrm{GeV}$. Surely, the leading term dominates at the colliders RHIC and LHC with available energies of hundreds and thousands $\mathrm{GeV}$.

All terms of Eq. (3) are positive at $\sqrt{s_{n n}}>3 \mathrm{GeV}$ and the leading term dominates at $\sqrt{s_{n n}}>6 \mathrm{GeV}$. These energies are below those at NICA and FAIR. Thus the Racah formula predicts quite noticeable effect already at energies about $10 \mathrm{GeV}$. In particular, the values of the cross section for $\mathrm{PbPb}$ collisions are $1.4 \mathrm{~kb}$ at $\sqrt{s_{n n}}=10 \mathrm{GeV} ; 22.8 \mathrm{~kb}$ at $100 \mathrm{GeV}$ and 97.5 $\mathrm{kb}$ at $1 \mathrm{TeV}$. The small mass of electrons defines so large values of the cross sections.

This formula was confirmed also by considering the Feynman diagrams with two photons emitted by colliding ions and producing the electronpositron pair. That is why the ultraperipheral collisions are often called as the two-photon processes. Correspondingly, the higher order corrections

\footnotetext{
${ }^{3}$ The form factors are usually accounted in the framework of the equivalent photon approximation.
} 
due to the additional photons emitted by ions were evaluated. These graphs are reproduced in many publications on this subject. The estimated part of the Coulomb correction to the Racah formula proportional to $l^{2}$ is negative [15, 16, 17, 18]:

$$
\sigma_{C}=-\frac{56}{9 \pi} \frac{Z^{4} \alpha^{4}}{m_{e}^{2}} f(Z) l^{2},
$$

where

$$
f(Z)=(Z \alpha)^{2} \sum_{n=1}^{\infty} \frac{1}{n\left(n^{2}+(Z \alpha)^{2}\right)} .
$$

It is negligibly small for protons but becomes essential for heavy ions. Its account for $\mathrm{Pb}$ ions leads to the replacement of the factor 8.3 in Eq. (3) by 16 approximately. The preasymptotical behavior of the cross section changes. The above estimates at different energies should be corrected correspondingly. At $10 \mathrm{GeV}$ the cross section becomes more than twice smaller. The estimates are not reliable enough because the term $l^{3}$ is not dominating anymore. Even at the LHC energies the cross section becomes smaller by about $13 \%$. Energies of NICA and FAIR are close to the threshold.

The unitarity corrections accounting for the loops of the light-by-light scattering in Feynman diagrams are small for the ultraperipheral graphs of the electron-positron production. In distinction, the Coulomb correction becomes much less for muon pairs production while the role of the unitarity corrections increases [19. At the same time, these conclusions and quantitative estimates of the cross section values according to Eqs (3) and (44) can change with account of the nuclear form factors [20]. These problems are crucial in connection with the so called ultraperipherality parameter introduced below. It is related to the numerical factors discussed above.

The multiple pair production was also estimated by different theoretical methods (see, e.g., [15, 16, 12]). The multiple pair production happens to be even more active at small impact parameters than the creation of a single pair. However, the total cross section is not very sensitive to small impact parameters. The main contribution comes from large impact parameters. Therefore the single pairs dominate.

\subsection{The differential distributions}

The precise differential distributions of the electron-positron pairs produced in the two-photon collisions are rather complicated. They contain 20 indepen- 
dent helicity amplitudes [21]. Matrix elements of the perturbative approach squared become strongly intermixed in the differential distributions. Some simplified expressions are written in the review [8]. As an example, we show the leading term of the distribution of the mass $W$ of the produced $e^{+} e^{-}$ system (Eq. (5.27) in [8]):

$$
\frac{d \sigma}{d W^{2}}=\frac{2\left(Z_{1} Z_{2} \alpha\right)^{2} \sigma_{\gamma \gamma \rightarrow e^{+} e^{-}}\left(W^{2}\right)}{3 \pi^{2} W^{2}} \ln ^{3} \frac{\left(p_{1} p_{2}\right)}{M_{1} M_{2}} .
$$

The detailed studies of the characteristics of the dilepton pairs production is still at the very initial stage. Their analysis shows that the dominant contribution to the total cross section at relativistic energies is provided by the region of production of electrons at small angles, small transverse

momenta and small pseudorapidities of the pain 4 . Therefore, the photons with small squared 4-momenta (virtualities) are most important. They can be considered as being almost real (massless). Then the differential cross section can be approximated by a product of the total cross section of the $\gamma \gamma$ transition to the electron-positron pair and the differential fluxes of photons which appeared already in Fermi's papers [1, 2]. Herefrom the equivalent photon approximation (see Section 4) follows [3, 4]. The maximum photon energy $\omega_{\max }=\sqrt{s_{n n}} / m b$ increases at higher collision energies and becomes smaller at large impact parameters.

The formulas (11) and (6) are valid for the point-like source of the electromagnetic radiation. The charge distribution inside the colliding protons and heavy nuclei must be taken into account. The intensity of the photon fields depends on the transverse distance (the impact parameter $b$ ) between the centers of the colliding nuclei. Therefore their radii $R_{i}$ enter the game due to the requirement $b>R_{1}+R_{2}$. These problems are considered within the equivalent photon approximation.

\subsection{Bound-free processes}

Before delving into these problems, let us mention the so called bound-free effect induced by the creation of the electron-positron pairs. This name is used when the created electron is captured by one of the ions while the positron flyes away. This is an important source of beam ions loss. The

\footnotetext{
${ }^{4}$ The problem of the widening of these distributions compared to their expressions in the perturbative approach is considered in the recent paper [22].
} 
charge-to-mass-ratio $Z / A$ changes and new ions do not follow the former trajectory. This loss puts some limits on luminosity. Such ions can damage the accelerator magnets, beam pipes and even its external safety walls at the distances of hundreds meters. They deposit their energy in a localized region of the beam pipe and heat it. That is of practical importance for operation of accelerators in the heavy-ion modes. The capture cross section is higher for more heavy ions. However, the cross section of these processes increases with energy increase only logarithmically [9, 23], i.e. slower than the main process which rises as the cube of the logarithm. The total cross section of the ultraperipheral collisions of lead nuclei can be as large as $200 \mathrm{~kb}$ at the LHC while the capture cross section is about $200 \mathrm{~b}$. The pair production with capture will become comparable with the production of free pairs at lower energies of NICA and FAIR.

\section{The equivalent photon approximation}

The essense of the equivalent photon approximation is already demonstrated by the Eq. (6). The Feynman diagrams of all processes with two-photon interactions contain a blob describing the transformation of these photons to some final states (e.g., $e^{+} e^{-}$considered above). Thus it can be described by the cross sections of these processes. The missing element of the whole picture are the photon fluxes in between the colliding charged objects. Namely they were the main purpose of Fermi's research as clearly stated in the citation put at the beginning of this review. The photons carrying small fractions $x$ of the nucleon energy dominate in these fluxes. The distribution of equivalent photons generated by a moving (point-like!) nucleus with the charge $Z e$ and carrying the small fraction of the nucleon energy $x$ integrated over the transverse momentum up to some value (see, e.g., [24]) leads according to the method of equivalent photons to the flux

$$
\frac{d n}{d x}=\frac{2 Z^{2} \alpha}{\pi x} \ln \frac{u(Z)}{x} .
$$

The ultraperipherality parameter $u(Z)$ depends on the nature of colliding objects and created states. Its physical meaning is the ratio of the maximum adoptable transverse momentum to the nucleon mass as the only massless parameter of the problem. In the perturbative approach it should incorporate the radiative corrections that change the preasymptotical dependence 
of the cross section (see comments after the Eq. (44)). It differs numerically in various approaches [8, 25, 26, 27, 28, 29, 30, 31, 32, 33, 34. It depends on considered processes, as well as on charges $Z_{i} e$, sizes and impact parameters of colliding objects (form factors and absorptive factors). The impact parameters cannot be measured but, surely, should exceed the sum of the radii of colliding ions. Otherwise the strong (QCD) and photonuclear interactions enter the game. This requirement can be restated as a bound on the exchanged transverse momenta, such that the objects are not destroyed but slightly deflected by the collision and no excitations or nuclear transitions happen. The bound depends on their internal structure, i.e., on forces inside them. These forces are stronger for a proton than for heavy nuclei. Therefore protons allow larger transverse momenta. The quantitative estimates of the parameter $u$ for different processes are obtained from comparison with experimental data and confronted with theoretical approaches described in more detail in the next section.

The equivalent photon approximation allows a clear separation into a purely kinematical effect of photon fluxes and the dynamical cross sections of their interactions. Besides the electron-positron pairs considered theoretically in Refs [5, 7] and observed, e.g., in Refs [35, 36], other pairs of oppositely charged particles with even $C$-parity can be created in the two-photon collisions. For example, pairs of muons produced in ultraperipheral collisions are observed at LHC [37, 38, 39, 40, 41]. The light-by-light scattering described theoretically by the loop of charged particles is also detected at LHC [42, 43, 44. Some neutral $C$-even bosons composed of quark-antiquark pairs can be produced in the two-photon interactions. This process is especially suitable for the compact theoretical demonstration [33] of the $\ln ^{3} \gamma$-law.

The exclusive cross section of the production of the resonance $R$ in the two-photon collisions of nuclei $A$ can be written as

$$
\sigma_{A A}(R)=\int d x_{1} d x_{2} \frac{d n}{d x_{1}} \frac{d n}{d x_{2}} \sigma_{\gamma \gamma}(R),
$$

where the fluxes $d n / d x_{i}$ for the colliding objects 1 and 2 are given by Equation (17) and (see Ref. [8])

$$
\sigma_{\gamma \gamma}(R)=\frac{8 \pi^{2} \Gamma_{t o t}(R)}{m_{R}} B r(R \rightarrow \gamma \gamma) B r_{d}(R) \delta\left(x_{1} x_{2} s_{n n}-m_{R}^{2}\right) .
$$

Here, $m_{R}$ is the mass of $R, \Gamma_{t o t}(R)$ its total width and $B r_{d}(R)$ denotes the branching ratio to a considered channel of its decay. $s_{n n}=(2 m \gamma)^{2}, m$ is 
a nucleon mass. The $\delta$-function approximation is used for resonances with small widths compared to their masses. The resonance is registered according to the peak in the distribution of the effective mass of the decay products $\sqrt{x_{1} x_{2} s_{n n}}$. As you see, the perturbative matrix element approach is replaced in the equivalent photon approximation by the semiclassical probabilistic scheme accounting for the structure of Feynman diagrams.

The integrals in Eq. (8) can be easily calculated so that one gets the analytical formula

$$
\sigma_{A A}(R)=\frac{128}{3} Z^{4} \alpha^{2} B r(R \rightarrow \gamma \gamma) B r_{d}(R) \frac{\Gamma_{t o t}(R)}{m_{R}^{3}} \ln ^{3} \frac{2 u m \gamma}{m_{R}}
$$

The asymptotical $\ln ^{3} \gamma$ behavior is valid again. The factor $2 m u / m_{R}=1 / \gamma_{0}$ defines the preasymptotical behavior of the ultraperipheral cross section of production of the resonance $R$. The structure of this formula is similar to that used for $e^{+} e^{-}$production (3). The variations of the parameter $u$ can account for the subleading terms proportional to $\ln ^{2} \gamma$. The asymptotical limit is reached at

$$
\gamma \gg m_{R} / 2 u m
$$

where the terms increasing slower than $\ln ^{3} \gamma$ can be neglected.

The parameter $u$ can be found from Eq. (10) if the exclusive cross sections of the ultraperipheral production of $\pi^{0}$-mesons or parapositronium are measured. The analogous formulae are obtained [45, 46] for the creation of the $C$-odd states like $\rho^{0}$-mesons or orthopositronium.

\section{The preasymptotical behavior of the cross sections}

The rapid asymptotical increase of the total cross section of ultraperipheral collisions as $\ln ^{3} \gamma$ poses the question about its comparison with the total cross section of purely hadronic interactions which can not increase stronger than $\ln ^{2} \gamma$ according to the Froissart theorem [47] stemming from general theoretical principles. According to experimental data on proton-proton collisions their increase is even slower at present energies.

The cross section for a single neutral pion production in ultraperipheral collisions of two protons according to (10) is compared in Ref. [48] with experimental data at $\mathrm{TeV}$ energies on the corresponding production channel 
in strong interactions. This cross section is about $1 \mathrm{nb}$, while single $\pi^{0} \mathrm{~s}$ are produced in strong interactions with cross sections of the order of $0.3 \mathrm{mb}$. It is shown that the additional $\ln \gamma$ factor is not large enough and absolutely insufficient for such ultraperipheral processes to dominate in proton-proton collisions over strong forces at any realistic energies. The background for $\pi^{0}$ production due to strong interactions at small impact parameters must be enormously large. Some special cuts should be imposed to separate the ultraperipheral events. The specific kinematics of ultraperipheral processes can be used for such cuts as shown in Section 7 .

The analogous estimates for collisions of heavy nuclei are more complicated due to the lack of the information about the definite reaction channels. One can just state that the large numerical factor $Z^{4} / A^{2 / 3} \approx 10^{6}$ in the ratio of the ultraperipheral to purely nuclear (strong) interactions would favour heavy nuclei compared to protons.

Another preasymptotical problem is related to the energy behavior of the ultraperipheral cross sections in the lower energy region. The Racah formula written as (3) clearly demonstrates the $\ln ^{3} \gamma$ asymptotics and shows that the numerical factor 8.3 determines the preasymptotical behavior of the ultraperipheral cross section. Moreover it is modified by the radiative corrections. This numerical factor transforms in the equivalent photon approximation to the ultraperipherality parameter $u(Z)$ which has a meaning of the ratio of maximum adoptable transferred momentum to the nucleon mass. This parameter incorporates the form factors and the impact parameters suppression. It influences the estimates of the cross sections, especially at lower energies. That becomes important, for example, at energies of NICA and FAIR. The electron-positron pair (as well as para- and ortho-positronium) production seems to be feasible there [48] if the optimistic results from studies at the LHC [34, 50, 51] are taken into account and extrapolated. The $\pi^{0}$-production asks for energies close to those of NICA and is very sensitive to the estimates of the parameter $u$ shown in Section 7 .

\section{Theoretical analysis of exclusive dilepton production}

The perturbative matrix element approach (11), (4) clearly demonstrates that the higher order corrections can change the preasymptotical values of the cal- 
culated cross section by changing the numerical factor in the argument of the $\mathrm{ln}^{3}$-term. Its predictive power suffers also from the consideration of colliding objects in Feynman diagrams as the structureless point-like particles. This deficiency can be partially avoided within the equivalent photon approximation (8) by the insertion and the interpretation of the ultraperipherality parameter $u(Z)$.

To take into account the size and the electric charge distribution inside the colliding hadrons and ions one must somewhat generalize the Eq. (8).

The density of the photon flux depends on the structure of colliding objects and on their impact parameter 5 . In terms of the form factors $F$ of colliding objects it looks like [10, 34, 53]:

$$
\frac{d^{3} n}{d^{2} b d x}=\frac{Z \alpha}{\pi^{2} x}\left[\int d q_{\perp} q_{\perp}^{2} J_{1}\left(b q_{\perp}\right) \frac{F\left(q_{\perp}^{2}+m^{2} x^{2}\right)}{q_{\perp}^{2}+m^{2} x^{2}}\right]^{2} .
$$

The electromagnetic fields of relativistic ions look like the narrow pancakes perpendicular to their trajectories and moving together with ions. Their interaction can be approximated by the $\delta$-function in this plane at small $x<0.1 / m b$ [27]. It is strongest when the ions come close to one another. However, the impact parameter between them should be larger than the sum of their radii. Otherwise they get involved in strong interactions, and huge background to the products of ultraperipheral interactions appears. To exclude such collisions, the cutoff factor $P\left(\left|\mathbf{b}_{1}-\mathbf{b}_{2}\right|\right)$ is introduced. It is determined by soft physics with low transferred momenta. Therefore it is non-perturbative and phenomenological. If the colliding ions are considered as the black disks, this factor forbids completely the impact parameters smaller than the sum of ions radii and is written in the following way

$$
P=\theta\left(\left|\mathbf{b}_{1}-\mathbf{b}_{2}\right|-R_{1}-R_{2}\right)
$$

where $R_{i}$ are their radii.

The Eq. (8) is generalized 6 as

$$
\sigma_{A A \rightarrow A A X}=\int d x_{1} d x_{2} d^{2} b_{1} d^{2} b_{2} \frac{d^{3} n}{d^{2} b_{1} d x_{1}} \frac{d^{3} n}{d^{2} b_{2} d x_{2}} \sigma_{\gamma \gamma \rightarrow X} P\left(\left|\mathbf{b}_{1}-\mathbf{b}_{2}\right|\right),
$$

\footnotetext{
${ }^{5}$ The flux in Eq. (7) is integrated over the transverse momentum and the impact parameter.

${ }^{6}$ The symbol $R$ must be replaced by $X=l^{+} l^{-}$for dileptons. The cross section $\sigma_{\gamma \gamma \rightarrow l^{+} l^{-}}$ is given by the Breit-Wheeler formula $[52$.
} 
The choice of the cutoff factor determines the ultraperipherality parameter $u(Z)$. If the heavy ions stay intact after the collision then the Eq. (13) should be used. At smaller impact parameters they do not survive. The hope that the form factors in Eq. (12) satisfy this requirement automatically is hardly realistic. The photon flux computed in Ref. [50] for $\mathrm{PbPb}$ interactions at the energy $5.02 \mathrm{GeV}$ per nucleon pair (see Fig. 3a in Ref. [50]) becomes much smaller if the additional cut according to (13) is imposed on it even for the realistic form factor. For processes with initial protons, the elastic scattering at small impact parameters can be taken into account. The spatial distribution of their inelastic profile depends on the collision energy [54]. The cutoff factor can be generalized either by the account of the proton opacity, as proposed in [55], or by the Glauber modification of (13), as proposed in [50]. The suppression factor $S^{2}$ has been used for the quantitative estimate of the cutoff factor on the cross section of the ultraperipheral processes:

$$
S^{2}=\frac{\iint d^{2} b_{1} d^{2} b_{2} \frac{d^{3} n}{d^{2} b_{1} d x_{1}} \frac{d^{3} n}{d^{2} b_{2} d x_{2}} P\left(\left|\mathbf{b}_{1}-\mathbf{b}_{2}\right|\right)}{\int_{b_{1}>0} \int_{b_{2}>0} d^{2} b_{1} d^{2} b_{2} \frac{d^{3} n}{d^{2} b_{1} d x_{1}} \frac{d^{3} n}{d^{2} b_{2} d x_{2}}}
$$

Its evaluation depends on the choice of the lower limits of the impact parameters in the numerator (e.g., compare Eq. (7) in 53] and Appendix 3 in [34]). This is the main origin of disagreement in the different choices of the ultraperipherality parameter mentioned above $[8,25,26,27,28,29,30,31$, 32, 33, 34.

Let us note, however, that the formulas for the total cross sections considered above are useful for understanding the energy behavior of ultraperipheral processes and some general estimates but they are not very practical in the direct applications to experimental results. The experimentally measured phase space volume is usually significantly smaller than the total one. The detector structure and possible backgrounds reduce it. The so-called fiducial cross sections taking into account these "cavities" and the requirement of the "ultraperipherality" are measured. The Monte Carlo generators, e.g., STARlight [56] or SuperChic [57, are often used both for the selection of the most favorable and admissible conditions and for the further comparison with experimental results. The corresponding cuts are imposed on the matrix elements or on the formulas, derived according to the equivalent photon approximation, for the computation of the fiducial cross sections, i.e., those which account for experimental cuts. The advantage of the equivalent photon approximation compared to the perturbative calculations or the di- 
agram approach is that the analogous calculations can be done analytically (up to computing some simple integrals). Thus one gets the possibility to compare different approaches and parametrizations with experimental data and control the accuracy of the equivalent photon approximation. These experimental cuts were taken into account in the considered below papers [34, 50, 51].

The more general problem is related to the spatio-temporal inhomogeneities of the considered electromagnetic fields. They can play a prominent role in the production of secondary particles. In particular, it is shown in [58] that it can result in the increased number of soft photons, i.e. in higher photon fluxes. Nonlinear effects of strong-field QED are related to the string problem [59] and can become important in heavy-ion collisions. In particular, the dynamically assisted Schwinger effect and the Franz-Keldysh effect are considered in [60]. They could be studied with a proper combination of ultraperipheral fields and laser fields.

\section{Comparison with experimental data}

Prediction of large cross sections of ultraperipheral heavy ion collisions stimulated their experimental studies at RHIC [35] and LHC [36, 37, 38, 39, 40, 41]. There are special signatures of these processes. The dilepton pairs in the final state have very small transverse momentum. There are two rapidity gaps that separate the intact very forward ions from the dilepton pair. From the theoretical side the main problem is the proper estimates of the photon fluxes, i.e. the evaluation of the parameter $u(Z)$.

Production of $e^{+} e^{-}$pairs in heavy-ion collisions was studied first at RHIC by STAR Collaboration [35] and then at LHC by ALICE Collaboration [36]. The obtained rapidity and invariant mass distributions for the exclusive $e^{+} e^{-}$ production by $\gamma \gamma$ interactions in $\mathrm{PbPb}$ collisions at $\sqrt{s}=2.76 \mathrm{TeV}$ were compared in Refs [50, 51] with theoretical results using the above formulas. Besides the rigid absorption factor of the black disks (13), its a'la Glauber model modification was considered. What concerns form factors, three of them were put on the trial: point-like, monopole and realistic which corresponds to the Fourier-transform of the Wood-Saxon charge-density distribution of the nucleus. The general behavior of both distributions is well reproduced by theoretical results except the region of small invariant masses below $2.3 \mathrm{GeV}$ (see Fig. 5 in [50]). The experimental cuts were imposed on the computed 
distributions. It was concluded that the modification of the absorption factor is unimportant. Both precise and monopole form factors fit experimental results at high masses rather well within error bars while the point-like one declines from them. The experimental distribution is higher than the theoretical one at small masses less than $2.3 \mathrm{GeV}$ in [50] but declines slightly only at that single point in [51] (see Fig. 3 there). The low masses become the most important ones at the lower energies of NICA and FAIR. This region must be carefully studied there.

Production of $\mu^{+} \mu^{-}$pairs in pp collisions was first observed in 1990 at CERN's Intersecting Storage Rings (ISR) [61]. However the detailed experimental studies [37, 39, 40, 41] and comparison with theory [34, 50, 51] became possible only recently.

The ATLAS data presented in Ref. [37] are confronted to theoretical results in Refs [50, 51] in the same manner as done above for $e^{+} e^{-}$data. Rapidity and invariant mass distributions for the exclusive $\mu^{+} \mu^{-}$production by $\gamma \gamma$ interactions in $\mathrm{PbPb}$ collisions at $\sqrt{s}=5.02 \mathrm{TeV}$ are plotted (see Fig. 4 in [50] and Fig. 4 in [51]). The agreement with theory is less satisfactory within the precision of experimental data than for electron-positron pairs, especially for the case of the point-like form factor, as expected.

The very detailed comparison with experiment is done in the paper [34]. It helped to get more definite information on the parameter $u(Z)$. The experimental results from pp collisions at $13 \mathrm{TeV}$ [39] and $\mathrm{PbPb}$ collisions at $5.02 \mathrm{TeV}$ per nucleon pair [40] were considered.

The total cross section of the ultraperipheral production of muon pairs in the equivalent photon approximation is

$$
\sigma\left(Z Z(\gamma \gamma) \rightarrow Z Z \mu^{+} \mu^{-}\right)=\frac{28}{27} \frac{Z^{4} \alpha^{4}}{\pi m_{\mu}^{2}} \ln ^{3} \frac{u^{2} s_{n n}}{4 m_{\mu}^{2}} .
$$

Let us note that the energy dependence in Eqs. (10) and (16) is the same for $m_{R}=2 m_{\mu}$ as expected. The preasymptotical behavior is determined by the factor $u$.

The cuts on the invariant mass of the $\mu^{+} \mu^{-}$pair (on the fraction $x$ in Eq. (17)), on the muon transverse momentum (on the differential $p_{T}$-distribution of $\gamma \gamma$ processes) and on the pseudorapidity (required by the detector geometry) were imposed on Eq. (14) both for pp-collisions at $13 \mathrm{TeV}(Z=1)$ and for $\mathrm{PbPb}$-collisions at $5.02 \mathrm{TeV}$ per nucleon pair. The corresponding integrals are easily computed. These cuts reduce drastically the cross section values. 
For example, for pp-processes the value $0.22 \mu \mathrm{b}$ according to Eq. (16) is reduced to $3.35 \mathrm{pb}$. If corrected for absorptive effects [53] it gives $3.06 \pm 0.05 \mathrm{pb}$. The chosen cuts coincide with those imposed in studies of the ATLAS collaboration [39] which measured the value $3.12 \pm 0.07$ (stat.) \pm 0.10 (syst.) pb. The SuperChic Monte Carlo program [57] which incorporates, in principle, both ordinary and ultraperipheral processes predicts $3.45 \pm 0.06 \mathrm{pb}$. Theoretical results are in agreement with experimental data and show that ultraperipheral processes dominate over other sources in this fiducial volume. Analogous conclusions were obtained for lead-lead collisions [34]. Here, due to $Z^{4}$ enhancement the measured fiducial cross sections are on the $\mu \mathrm{b}$ scale compared to pb's for pp-collisions.

The ultraperipherality parameter $u$ is the least precisely determined element of the equivalent photon approximation. As described above, its evaluation crucially depends on two main factors accounting for the impact parameter suppression $P(b)$ and for the charge distribution inside ions (form factors $F(q)$ ). The careful treatment of form factors of protons and nuclei with account of the photon virtuality (see also Refs [25, 27] where the problem was treated in more detail) and the suppression factors [34] lead to its values $u_{p p} \approx 0.2$ for $\mathrm{pp}$ and $u_{P b P b} \approx 0.02$ for $\mathrm{PbPb}$-collisions within the factors about 1.5 which depend on the particular shape of the form factors. At the very beginning, these values of the parameters $u(Z)$ were qualitatively estimated from general physics arguments and then confirmed by the successful comparison of theoretical predictions with experimental data.

It is remarkable that these values of the ultraperipherality parameter $u$ agree quite well with those obtained in Ref. [46] for the ultraperipheral cross sections of $\pi^{0}$-production according to Eq. (10) at RHIC and LHC energies. Their values (28 mb at LHC) shown in the Table of Ref. [46] easily lead to $u_{P b P b} \approx 0.013$. This value agrees up to the factor 1.5 with that shown above.

The knowledge of the ultraperipherality parameters helps to estimate possible effects at the lower energies of NICA and FAIR. They favor the ultraperipheral processes of $e^{+} e^{-}$and positronia production [48] there, while the $\pi^{0}$-production is questionable because the argument of the logarithm in Eq. (10) is close to 1 and, therefore, the non-leading terms similar to those in Eq. (3) must be taken into account (available near the threshold at the estimates of [34] and unavailable at the estimates of [46]) $)^{7}$.

\footnotetext{
${ }^{7}$ The earlier estimates [33 based on the extremely strong cuts of the impact parameters imposed in 32 produced the much higher threshold for such processes.
} 


\section{Searches for new physics}

The clean channel of $\gamma \gamma$ interactions in ultraperipheral collisions is often discussed in connection with searches for new physics. Before the Higgsboson discovery it was actively debated as one of the possible sources for its creation (see, e.g., [62, 63]). Nowadays, the main topics include searches for supersymmetric particles [62, 64, 65, 66, 67, 68, 69, 70, 71], magnetic monopoles [72, 73, 74, gravitons and possible extra spatial dimensions of the Kaluza-Klein theory with large compact dimensions in addition to the 4 dimensions of Minkowski spacetime [63, 75], axion-like pseudoscalar particles [76, 77, 78, 79, 80, 81, 82], which would induce anomalous scattering of light-by-light, radions [83], unparticles [84], impact of supersymmetry on properties of the observed particles (e.g., the virtual sparticles in Feynman diagrams for the anomalous magnetic moment of the tau lepton [85]). There are no experimental signatures of these effects yet. Some lower limits, e.g., on the masses of supersymmetric particles [86] or on the masses of the spin 0,1/2, or 1 Dirac monopoles [73] are established.

The very detailed proposal of searches for the supersymmetric particles in ultraperipheral proton-proton collisions at the LHC was presented recently in the Ref. [71]. It is proposed to search for charginos 8 with masses about 100 $\mathrm{GeV}$. Even though the LHC results exclude within a large interval of theoretical parameters their production with masses below $1 \mathrm{TeV}$ according to [86], this conclusion is not valid in the case when the masses of lightest chargino and lightest neutralino are approximately equal. Namely this rather exotic possibility with the lightest chargino somewhat heavier than the lightest neutralino is studied. The leading order Feynman diagrams for chargino pairs production in ultraperipheral collisions look similar to those for production of the electron-positron pairs considered above. The estimates of the total cross sections give the values $2.84 \mathrm{fb}$ for pp collisions at $13 \mathrm{TeV}$ and $21.2 \mathrm{pb}$ for $\mathrm{PbPb}$ collisions at $5.02 \mathrm{TeV}$ per nucleon. If both protons are required to be registered in forward detectors 9 and the experimental cuts similar to the described above cuts for muon pairs data are imposed, the fiducial cross section in proton-proton processes is reduced to $0.72 \mathrm{fb}$. The background due to muon pairs and pile-up is estimated. It is shown that the peaks from

\footnotetext{
${ }^{8}$ Two charginos and four neutralinos are the supersymmetric partners of the electroweak bosons.

${ }^{9}$ It allows strong background suppression and complete reconstruction of event kinematics.
} 
chargino and muons are well separated and the chargino peak is clearly visible over the pile-up if the special cut on the longitudinal momentum of the final state system is used (see Fig. 6 in Ref. [71]).

\section{Conclusions}

The ultraperipheral processes provide very important information about strong electromagnetic fields. Experimental data on dilepton production are successfully described by the equivalent photon approximation. The interaction of high energy photons in the dense electromagnetic clouds surrounding relativistic protons and heavy ions opens ways to studies of new physics in the processes of production of new objects. The theoretical methods of their description are well developed and prove their applicability when compared with experimental data.

\section{Acknowledgement}

This work was supported by the RFBR project 18-02-40131 and RANCERN program.

\section{References}

[1] Fermi E Z. Physik 29315 (1924)

[2] Fermi E Nuovo Cim. 2143 (1925), translated from Italian by M. Gallinaro and S. White in arXiv:hep-th/0205086

[3] Weizsäcker C F V Z. Physik 88612 (1934)

[4] Williams E J Phys. Rev. 45729 (1934)

[5] Landau L D, Lifshitz E M Phys. Z. Sowjetunion 6244 (1934)

[6] Anderson C D Phys. Rev. 43491 (1933)

[7] Racah G Nuovo Cimento 1493 (1937)

[8] Budnev V M et al. Phys. Rep. 15181 (1975)

[9] Bertulani C A, Baur G Phys. Rep. 163299 (1988) 
[10] Baur G et al. Phys. Rep. 364359 (2002)

[11] Bertulani C A, Klein S K, Nystrand J Ann. Rev. Nucl. Part. Sci. 55 271 (2005)

[12] Baur G, Hencken K, Trautmann D Phys. Rep. 4531 (2007)

[13] Ruffini R, Vereshchagin G, Xue S Phys. Rep. 4871 (2010)

[14] Di Piazza A et al. Rev. Mod. Phys. 841177 (2012)

[15] Ivanov D Y, Schiller A, Serbo V G Phys. Lett. B 454155 (1999)

[16] Lee R N, Milstein A I, Serbo V G Phys. Rev. A 65022102 (2002)

[17] Gevorkyan S R, Kuraev E A J. Phys. G 291227 (2003)

[18] Baltz A J Phys. Rev. C 71024901 (2005)

[19] Hencken K, Kuraev E A, Serbo V G Phys. Rev. C 75034903 (2007)

[20] Baltz A J Phys. Rev. C 80034901 (2009)

[21] Carlson C E, Tung W K Phys. Rev. D 6147 (1972)

[22] Klein S R et al. arXiv:2003.02947

[23] Meier H et al. Phys. Rev. A 63032713 (2001)

[24] Berestetsky V B, Lifshitz E M, Pitaevsky L P Kvantovaya Electrodinamika; Fizmatlit: Moscow, Russia, 2001.

[25] Baur G, Bertulani C A Z. Phys. A 33077 (1988)

[26] Klein S R, Nystrand J Phys. Rev. Lett. 842330 (2000)

[27] Guclu et al. Annals Phys. 2727 (1999)

[28] Baltz A J et al. Phys. Rev. C 80044902 (2009)

[29] Klusek-Gawenda M, Szczyrek A Phys. Lett. B 763416 (2016)

[30] Sengul et al. Eur. Phys. J. C 76428 (2016)

[31] Zha et al. Phys. Lett. B 789238 (2019) 
[32] Khoze V A, Martin A D, Ryskin M G arXiv:1902.08136

[33] Dremin I M Int. J. Mod. Phys. A 341950068 (2019)

[34] Vysotsky M I, Zhemchugov E V Phys. Usp. 189975 (2019)

[35] Adams J et al. [STAR Collab.] Phys. Rev. C 70031902 (2004)

[36] Abbas E et al. [ALICE Collab.] Eur. Phys. J C 732617 (2013)

[37] Dyndal M [ATLAS Collab.] Nucl. Phys. A 967281 (2017)

[38] Khachatryan V et al [CMS Collab.] Phys. Lett. B 772489 (2017)

[39] Aaboud M et al. [ATLAS Collab.] Phys. Lett. B 777303 (2018)

[40] Aaboud M et al. [ATLAS Collab.] ATLAS-CONF-2016-025. 2016.

[41] Arratia M arXiv:1611.05145

[42] Aaboud M et al. [ATLAS Collab.] Nat. Phys. 13852 (2017)

[43] d'Enterria D et al. [CMS Collab.] Nucl. Phys. A 982791 (2019)

[44] Aad G et al. [ATLAS Collab.] Phys. Rev. Lett. 123052001 (2019)

[45] Kotkin G L et al. Phys. Rev. C 592734 (1999)

[46] Bertulani C A, Navarra F Nucl. Phys. A 703861 (2002)

[47] Froissart M Phys. Rev. 1231253 (1961)

[48] Dremin I M Universe 64 (2020)

[49] Klein S R Phys. Rev. C 97054903 (2018)

[50] Azevedo C, Goncalves V F, Moreira B D Eur. Phys. J. C 79432 (2019)

[51] Szczurek A arXiv:1810.06249

[52] Breit G, Wheeler J A Phys. Rev. 461087 (1934)

[53] Dyndal M, Schoeffel L Phys. Lett. B 74166 (2015)

[54] Dremin I M Physics 133 (2019) 
[55] Harland-Lang L A, Khoze V A, Ryskin M G JHEP 03182 (2016)

[56] Klein S R et al. Comput. Phys. Commun. 212258 (2017)

[57] Harland-Lang L A, Khoze V A, Ryskin M G Eur. Phys. J. C 7939 (2019)

[58] Aleksandrov I A, Plunien G, Shabaev V M Phys. Rev. D 100116003 (2019)

[59] Fradkin E S, Tseytlin A A Phys. Lett. B 163123 (1985)

[60] Taya H Phys. Rev. D 99056006 (2019)

[61] Antreasyan D et al, Preprint CERN-EP/80-82 (1990)

[62] Vidovic M, Greiner M, Soff G Phys. Rev. C 472288 (1993)

[63] Piotrzkowski K Phys. Rev. D 63071502 (2001)

[64] Ohnemus J, Walsh T F, Zerwas P M Phys. Lett. B 328369 (1994)

[65] Schul N, Piotrzkowski K Nucl. Phys. Proc. Suppl. 179289 (2008)

[66] Khoze V A et al. Eur. Phys. J. C 68125 (2010)

[67] Harland-Lang L A et al. Eur. Phys. J. C 721969 (2012)

[68] Khoze V A, Martin A D, Ryskin M G J. Phys. G 44055002 (2017)

[69] Harland-Lang L A et al. JHEP 1904010 (2019)

[70] Beresford L, Liu J Phys. Rev. Lett. 123141801 (2019)

[71] Godunov S I et al. JHEP 01143 (2020)

[72] 't Hooft G Nucl. Phys. B 79276 (1974)

[73] Abbott B. et al. [D0 Collab.] Phys. Rev. Lett. 81524 (1998)

[74] Acharya B et al. Phys. Lett. B 782510 (2018)

[75] Abers S C, Norbury J W, Poyser W J Phys. Rev. D 62116001 (2000)

[76] Knapen S et al. Phys. Rev. Lett. 118171801 (2017) 
[77] Bruce R et al. arXiv:1812.07688

[78] Baldenegro C et al. Phys. Lett. B 795339 (2019)

[79] Shaken S, Marsh D E, Xue S arXiv:2002.06023

[80] Coelho C et al. arXiv:2002.06027

[81] Sikivie P arXiv:2003.02206

[82] Inan S C, Kisselev A V arXiv:2003.01978

[83] Richard F arXiv:1712.06410

[84] Cakir O, Ozansoy K O Eur. Phys. J. C 56279 (2008)

[85] Beresford L, Liu J arXiv:1908.05180

[86] Tanabashi M et al. [Particle Data Group] Phys. Rev. D 98030001 (2018) 